\title{
LA-UR. 0904938
}

Approved for public release;

distribution is unlimited.
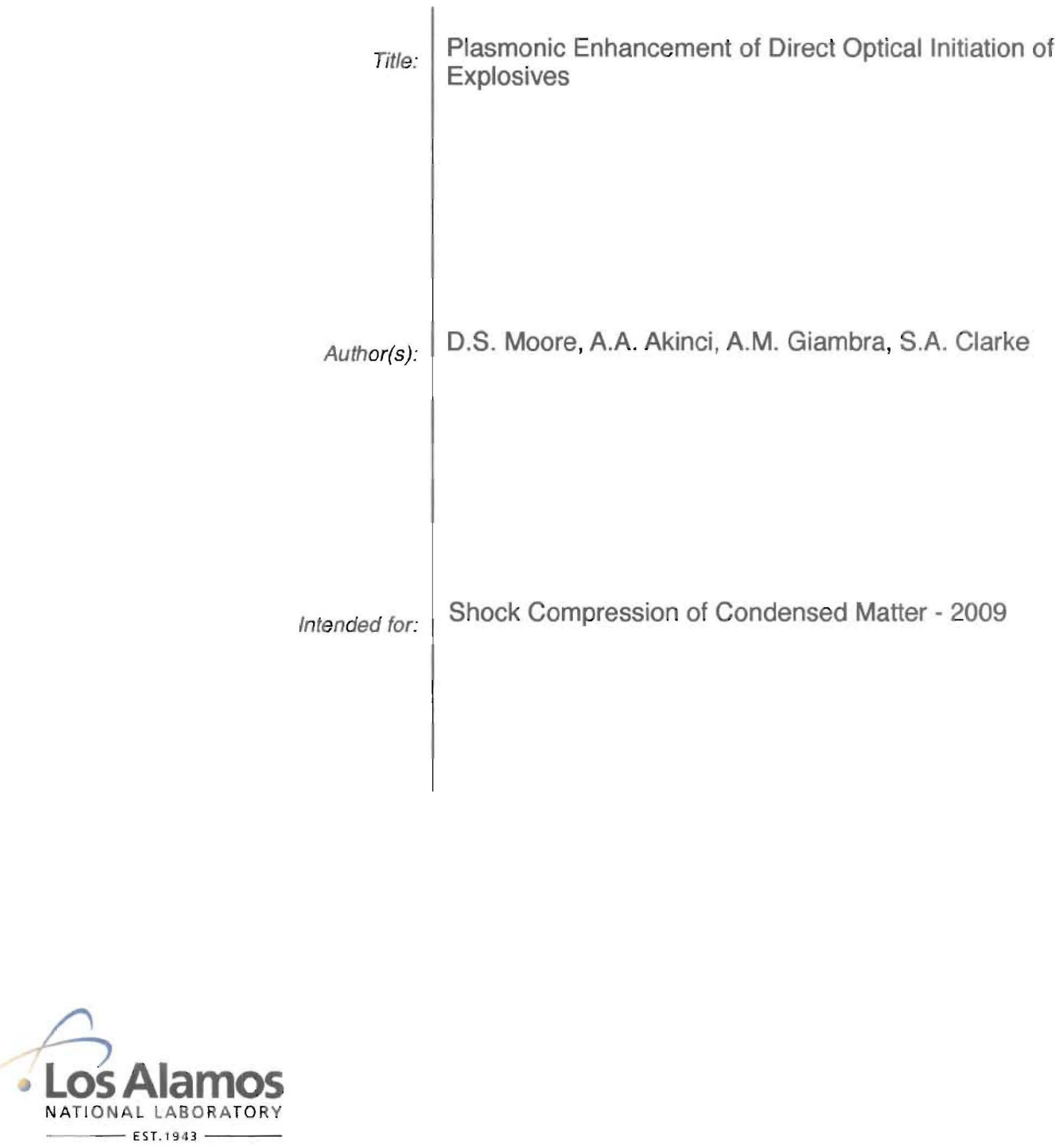

Los Alamos National Laboratory, an affirmative action/equal opportunity employer, is operated by the Los Alamos National Security, LLC for the National Nuclear Security Administration of the U.S. Department of Energy under contract DE-AC52-06NA25396. By acceptance of this article, the publisher recognizes that the U.S. Government retains a nonexclusive, royalty-free license to publish or reproduce the published form of this contribution, or to allow others to do so, for U.S. Government purposes. Los Alamos National Laboratory requests that the publisher identify this article as work performed under the auspices of the U.S. Department of Energy. Los Alamos National Laboratory strongly supports academic freedom and a researcher's right to publish; as an institution, however, the Laboratory does not endorse the viewpoint of a publication or guarantee its technical correctness. 



\title{
PLASMONIC ENHANCEMENT OF DIRECT OPTICAL INITIATION OF EXPLOSIVES
}

\author{
D.S. Moore, A.A. Akinci, A.M. Giambra, S.A. Clarke \\ Los Alamos National Laboratory, Los Alamos, NM $875+5$ USA
}

\begin{abstract}
Current Direct Optical Initiation (DOI) detonators use a laser focused onto a thin metal layer to drive a hot plasma and/or fragments into PETN powder. Previous studies showed a dramatic decrease in laser energies required to initiate the detonation using this approach over direct laser illumination of the PETN powder. Plasmonic metal nanostructures have been shown capable of strongly coupling laser energy into adjacent materials. We have incorporated gold nanospheres into PETN powder and are investigating their plasmonic enhancement of direct optical initiation via measurements of threshoid laser energies and streak camera measurements for calculation of run to detonation distances compared to other DOI schemes.
\end{abstract}

Keywords: Detonation, initiation, lasers, detonators, plasmonics

PACS: 42.65.Re, 42.62.Fi, 62.50.Ef, 82.40.Fp

\section{INTRODUCTION}

We are investigating the enhancement of direction optical initiation afforded by incorporation of plasmonic structures to amplify incident laser electric fields. Eversole, Luk'yanchuk and Ben-Yakar [1] have shown that gold nanospheres are able to dramatically reduce photoablation thresholds by enhancing the coupling of the laser into a dielectric material via surface plasmon resonances. They showed that the effect was not due to simple heating of the nanospheres, but rather amplification of the electric field. This was deduced from Atomic Force Microscope (AFM) images of the alignment and shape of the ablation craters, which were dumbbell in shape and aligned along the electric vector of the incident laser. The photoablation threshold was reduced up to 23 times from that needed without plasmonic enhancement. The studies described here were designed to determine whether this same phenomenology can be used to reduce the laser energy requirements for direction optical initiation of a detonator system involving PETN, a standard detonator explosive material.

\section{THEORY}

Following Eversole et al., the near-field scattering efficiency, $Q_{\mathrm{NF}}$, of a gold nanoparticle embedded in air as a function of radius can be expressed:

$Q_{\mathrm{NF}}=\frac{R^{2}}{\pi a^{2}} \int_{0}^{2 \pi} \int_{0}^{\pi} E_{s} E_{s}^{*} \sin \theta \mathrm{d} \theta \mathrm{d} \varphi$

where $R$ defines a spherical surface of which the integral is evaluated and $a$ is the particle radius. $Q_{\text {NF }}$ is a measure of the nanosphere's ability to convert the incident electric field intensity into a near-field intensity, and is proportional to the spare of the scattered electric field on the particle surface, Es, which is wavelength dependent and consists of all three components (angular and radial). The radial component (normal to the surface) dominates the scattered field, yielding large fields in the vicinity of the particle. 
For gold nanoparticles and an incident laser wavelength of $1064 \mathrm{~nm}$, the plot of $Q_{\mathrm{NF}}$ versus radius peaks near $250 \mathrm{~nm}$. Compared to smaller diameter gold nanospheres, the $250 \mathrm{~nm}$ particles also show greatly diminished absorption; from Mie calculations the scattered to absorbed energy ratio is expected to be greater than $600: 1$ making them excellent candidates to strongly couple incident laser radiation into detonator materials.

\section{EXPERIMENTAL}

\section{Sample preparation}

The nanosphere starting material is a $250 \mathrm{~nm}$ diameter gold colloid solution (Ted Pella Gold Colloid Product EM.GC250) in water. A slurry solution was made by adding 0.33 grams of PETN detonator powder (94-01-12) to 33 milliliters of the gold colloid solution and incorporating them homogeneously using a wrist shaker. A total of six batches/slurries were made. The slurries were shaken for approximately two hours and then allowed to air dry on a stirring stage with a small stir bar in each container to help keep the slurries homogeneous while drying.

\section{SEM analysis}

SEM imaging was performed using a JEOL7000F Field Emission Scanning Electron Microscope (FESEM), an ultra-high performance scanning electron microscope with a resolution 1.2 $\mathrm{nm}$ at $30 \mathrm{kV}$ and $3 \mathrm{~nm}$ at $1.0 \mathrm{kV}$. Random samples of the dried PETN/gold nanosphere slurry were placed on conductive carbon SEM pads and a 10 nm thick layer of $\mathrm{Au} / \mathrm{Pd}$ was applied using a model 208HR Cressington coater.

A representative SEM image is shown in Fig. I. The images were obtained in backscattering geometry, where the intensity also depends on atomic number so that the gold nanospheres show up as white spots. The image indicates little clustering and a reasonably even distribution. Multiple images were obtained and analyzed to obtain an average surface coverage of 0.035 particles $/ \mu \mathrm{m}^{2}$ and less than $4 \%$ clusters of two or more particles.
The apparent size of these nanospheres to the incident laser radiation is illustrated as in Figure 2, where the light grey disks are 23 times the diameter of the $\mathrm{Au}$ nanospheres $[1]$. The disks are drawn centered on each of the visible Au nanospheres, and indicate nearly half of the surface area is active with respect to laser radiation coupling to the PETN crystals.

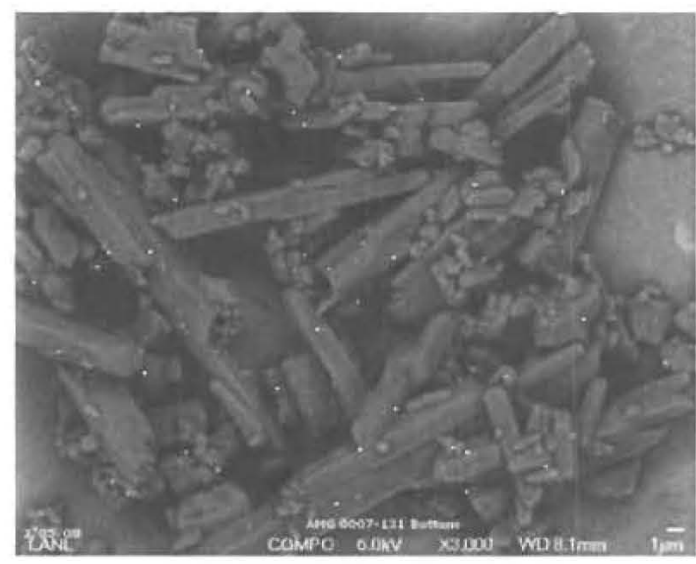

Figure 1. SEM image of the PETN/Au nanosphere material, taken in backscattering geometry. The $\mathrm{Au}$ nanospheres are visible as the white spots. (Image courtesy Ed Roemer, LANL).

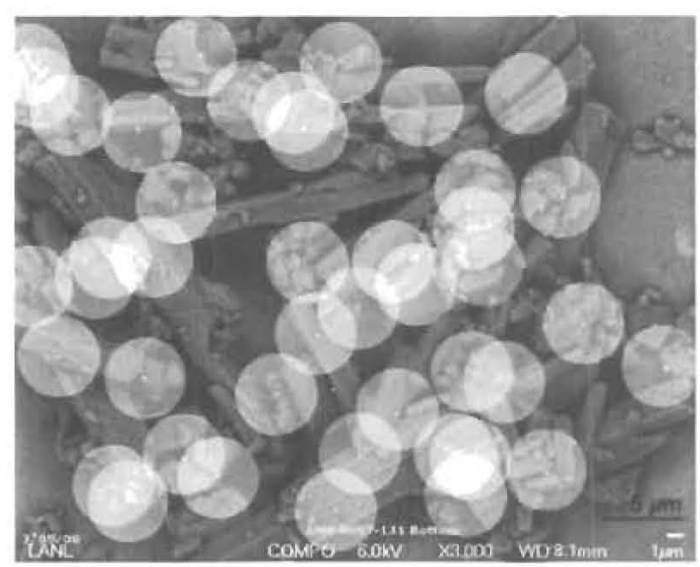

Figure 2. As Figure 1 with grey disks superimposed representing the plasmonic enhancement size for 1064 $\mathrm{nm}$ incident laser; each disk centered on a nanoparticle. 


\section{Sensitivity testing}

Standard Los Alamos material sensitivity release tests were performed on this PETN/Au nanosphere material, including Type 12 impact, spark, friction, vacuum stability, and DSC tests. All results were indistinguishable from the PETN starting material, as shown in Table 1.

\begin{tabular}{|l|l|l|}
\hline \multicolumn{1}{|c|}{ Test } & \multicolumn{1}{|c|}{ PETN/Au } & \multicolumn{1}{c|}{ PETN Ref. } \\
\hline Impact (type 12) & $15.2 \mathrm{~cm}$ & $14.9 \mathrm{~cm}$ \\
\hline Spark TIL & $0.0625 \mathrm{~J}$ & $0.0625 \mathrm{~J}$ \\
\hline Friction load & $7.6 \mathrm{~kg}$ & $8.2 \mathrm{~kg}$ \\
\hline DSC & & $140.8^{\circ} \mathrm{C}$ \\
\hline $\begin{array}{l}\text { Peak Melt } \\
\text { Endotherm }\end{array}$ & $140.5^{\circ} \mathrm{C}$ & $161.8^{\circ} \mathrm{C}$ \\
\hline $\begin{array}{l}\text { Decomposition } \\
\text { onset }\end{array}$ & $164.5^{\circ} \mathrm{C}$ & $205.0^{\circ} \mathrm{C}$ \\
\hline Peak Exotherm & $203.6^{\circ} \mathrm{C}$ & $0.3 \mathrm{~mL} / \mathrm{g}$ \\
\hline $\begin{array}{l}\text { Vacuum stability } \\
\text { (gas volume after } 48 \\
\text { hrat } 100^{\circ} \mathrm{C} \text { ) }\end{array}$ & $0.35 \mathrm{~mL} / \mathrm{g}$ & \\
\hline
\end{tabular}

Table 1. PETN/Au nanosphere material sensitivity test results. The PETN reference was Lot 94-01B

\section{DIRECT OPTICAL INITIATION TESTS}

To enable comparison with previous direct optical initiation results, the LANL designed and built ER-462 "Laser EBW" detonator was used [3]. These detonators have a $4 \mathrm{~mm}$ diameter by 2.54 $\mathrm{mm}$. length initial pressing of $0.88 \mathrm{~g} / \mathrm{cm}^{3}$ high surface are PETN. The output explosive is PETN at a density of $1.65 \mathrm{~g} / \mathrm{cm} 3$.

The laser energy was delivered via a 0.22 numericall aperture (NA) silica optical fiber (Polymicro FVP) with mechanically polished input and output faces. The $1064 \mathrm{~nm}$ laser pulses were optically coupled into the fibers and the output end was butted to the fused silica window at the optical end of the ER-462 initial pressing. The ER-462 as described is shown schematically in Fig. 3.

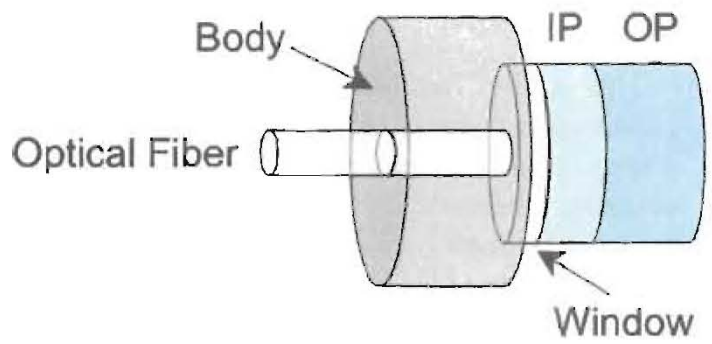

Figure 3. Schematic representation of the ER-462 "Laser EBW" detonator. IP is initial pressing and OP is output pellet.

\section{RESULTS}

Twelve separate experiments were performed for both the standard PETN initial pressing material and the PETN/Au nanosphere material. The Go / No Go data from each set was statistically evaluated using Excel to obtain the threshold laser energy, as is shown in Figure 4.

These results are very promising in that the improvement (reduction in threshold laser energy) provided by the Au nanospheres is more than a factor of two compared to the same PETN material without Au nanospheres. This improvement is comparable to that provided by the usually employed titanium layer on the silica window where it contacts the IP layer [2]

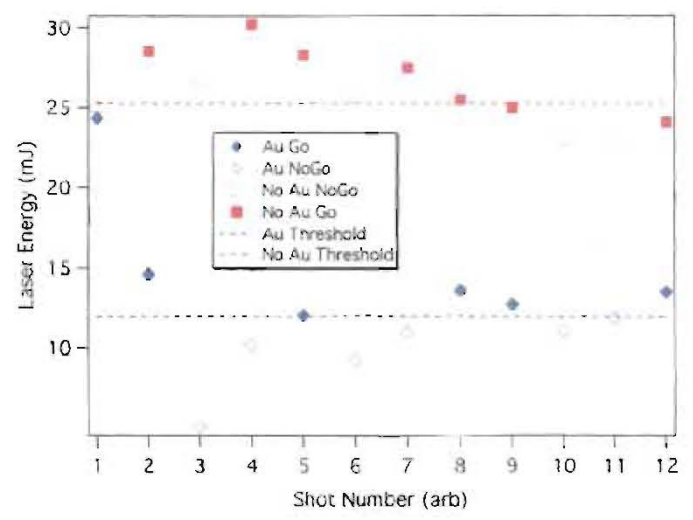

Figure 4. Go / No Go results for 12 laser EBW tests using standard PETN initial pressing compared to the results using the PETN/Au nanosphere material. 


\section{DISCUSSION}

This study has demonstrated that plasmonic enhancement does indeed strongly couple laser energy into energetic materials similarly to the coupling observed by Lukyanchuk et al. in their studies of plasmonically enhanced laser ablation of silicon surfaces [1]. The strong laser coupling leads to significant improvement in laser detonator thresholds. In addition, further improvements are possible as shown in Figure 2. The areal coverage afforded by these gold nanospheres can be increased at least a factor of two, which should improve the laser detonator thresholds the same factor. It remains unclear what thickness of the PETN/Au nanosphere material initial pressing is actually active during the laser initiation, so that further increases in gold nanosphere concentration might further improve the laser detonator thresholds. Given the effective scattering cross section for these nanoparticles, however, there is sure to be an asymptotic limit to the concentration. Further experiments are ongoing.

Also, another aspect of plasmonic enhancement has not yet been explored, that being the relative importance of plasmon resonance and plasmon scattering. This aspect can be explored using nanoshells and/or nanorods, where both the peak plasmon resonance and scattering wavelengths can be independently tuned [4]

\section{REFERENCES}

1. D. Eversole, B. Lukyanchuk, A. Ben-Yakar, Appl Phys. A 89:283-291 (2007)

2. M. Quinten, Appl. Phys. B 73:245 (2001)

3. A.A. Akinci, S.A. Clarke, K.A. Thomas, A.C. Munger, in Optical Technolgoes for Arming, Safing, Fuzing, and Firing II, W.J. Thomas, Jr, F.M Dickey, Eds. Proc. of SPIE Vol. 6287, paper 628709 (2006)

4. S.A. Love, B.J. Marquis, C.L. Haynes, Appl. Spectrosc. 62:246A-360A (2008)

\section{ACKNOWLEDGEMENTS}

We are indebted to Ed Roemer for his exacting SEM work, to Gabriel Avilucea for the material sensitivity data, and to the support staff at the Los Alamos Direct Optical Initiation Lab. The work described in this paper was performed at Los Alamos National Lab, operated by Los Alamos National Security, LLC for the National Nuclear Security Agency of the U.S. Department of Energy under contract DE-AC42-06NA25396. 